Research Article

\title{
High Neutrophil-to-Lymphocyte Ratio Predicts Hemorrhagic Transformation in Acute Ischemic Stroke Patients Treated with Intravenous Thrombolysis
}

\author{
Yong-Lin Liu $\mathbb{D}^{\mathrm{D}},{ }^{1}$ Jie-Kai Lu, ${ }^{1}$ Han-Peng Yin ${ }^{\mathbb{D}},{ }^{1}$ Pei-Shan Xia ${ }^{(D)},{ }^{1}$ Dong-Hai Qiu $\left(\mathbb{D},{ }^{1}\right.$ \\ Man-Qiu Liang $\left(1,,^{2}\right.$ Jian-Feng $Q u\left(\mathbb{1},{ }^{1}\right.$ and Yang-Kun Chen $\mathbb{( I )}^{1}$ \\ ${ }^{1}$ Department of Neurology, Dongguan People's Hospital (Affiliated Dongguan Hospital, South Medical University), Dongguan, \\ Guangdong Province, China \\ ${ }^{2}$ Department of Radiology, Dongguan People's Hospital (Affiliated Dongguan Hospital, South Medical University), Dongguan, \\ Guangdong Province, China
}

Correspondence should be addressed to Yang-Kun Chen; cykun78@163.com

Received 28 September 2019; Revised 22 December 2019; Accepted 6 January 2020; Published 28 February 2020

Guest Editor: Chao Li

Copyright ( 92020 Yong-Lin Liu et al. This is an open access article distributed under the Creative Commons Attribution License, which permits unrestricted use, distribution, and reproduction in any medium, provided the original work is properly cited.

Background. The relationship between the neutrophil-to-lymphocyte ratio (NLR) and hemorrhagic transformation (HT) in acute ischemic stroke (AIS) treated with intravenous thrombolysis (IVT) remains unclear. This study assessed whether high NLR is associated with HT in this population. Methods. Data were prospectively collected for continuous patients with AIS treated with IVT and retrospectively analyzed. Clinical variables included age, sex, vascular risk factors, National Institutes of Health Stroke Scale (NIHSS) score, onset-to-treatment time, and initial hematologic and neuroimaging findings. HT was confirmed by imaging performed within 3 days after IVT. Symptomatic HT (sHT) was defined as NIHSS score increased by 4 points compared with that on admission according to previously published criteria. The NLR value was based on the blood examination before IVT, and high NLR was defined as $\geq 75$ th percentile. Results. The study included 285 patients (201 (70.5\%) males, the mean age was 62.3 years (range 29-89)). Seventy-two (25.3\%) patients presented with HT, including three (1.1\%) with sHT. The median NLR was 2.700 (1.820-4.255, interquartile range). Seventy-one (24.9\%) patients had a high NLR ( $\geq 4.255)$ on admission. Univariate analysis indicated that patients with HT had higher NIHSS scores $(P<0.001)$, systolic blood pressure (SBP), platelet counts, lymphocyte counts, and NLR $(P<0.05)$, as well as a greater prevalence of high NLR than those without HT $(37.5 \%$ vs. $20.7 \%$ and $P=0.004)$. Patients with HT were more likely to have hypertension and AF. As lymphocyte counts and high NLR were highly correlated, we used two logistic regression models. In model 1 (with high NLR), NIHSS score on admission (odds ratio $(\mathrm{OR})=1.110,95 \%$ confidence interval $(\mathrm{CI})=1.015-1.044$, and $P=0.001), \mathrm{AF}(\mathrm{OR}=3.986,95 \%$ $\mathrm{CI}=2.095-7.585$, and $P<0.001)$, and high NLR $(\mathrm{OR}=2.078,95 \% \mathrm{CI}=1.078-4.003, P=0.029$, sensitivity 0.375 , and specificity 0.793 ) were significant predictors of HT. In model 2 (with lymphocyte counts), NIHSS score on admission (OR $=1.111,95 \%$ $\mathrm{CI}=1.050-1.175$, and $P<0.001), \mathrm{AF}(\mathrm{OR}=3.853,95 \% \mathrm{CI}=2.048-7.248$, and $P<0.001)$, and lymphocyte counts $(\mathrm{OR}=0.522$, $95 \% \mathrm{CI}=0.333-0.819$, and $P=0.005)$ were significantly associated with HT. Conclusions. High NLR could be a useful marker for predicting HT in AIS patients after IVT.

\section{Introduction}

Intravenous thrombolysis (IVT) with recombinant tissue plasminogen activator ( $\mathrm{r}$-tPA) is an effective treatment for acute ischemic stroke (AIS) when administered within the hyperacute period $[1,2]$. Hemorrhagic transformation (HT) is common in AIS with an incidence ranging from $8.5 \%$ to $40 \%$ [3-5], and symptomatic HT (sHT) is a risk factor for poor prognosis after AIS [6]. IVT has been reported to increase the incidence of HT markedly [7]. Atrial fibrillation (AF) $[8,9]$, National Institutes of Health Stroke Scale (NIHSS) score [10], blood glucose level [11], leukoaraiosis 
[12], dual antiplatelet agent treatment before IVT [13], and systolic blood pressure variability [14] are the predictors of HT after IVT.

In recent years, researchers have attempted to identify convenient serum biomarkers to help predict AIS outcomes. Several studies reported that a high neutrophil-to-lymphocyte ratio (NLR) was predictive of HT in AIS patients $[15,16]$. However, there were limited data on the relationship between NLR and HT in AIS patients treated with IVT. The present study was performed to assess whether high NLR is associated with HT in AIS patients after IVT.

\section{Methods}

2.1. Patients. AIS patients treated with IVT after admission to Dongguan People's Hospital between 1 January 2016 and 31 May 2019 were continuously recruited. The inclusion criteria were as follows: (1) age > 18 years, (2) AIS confirmed by magnetic resonance imaging (MRI), and (3) onset of stroke symptoms within 4.5 hours and treated with r-tPA. The exclusion criteria were as follows: (1) hemorrhagic lesions detected on initial computed tomography (CT), (2) temporary or permanent contraindications for MRI scan, (3) no acute lesion on diffusion-weighted imaging (DWI), and (4) additional endovascular therapy after IVT. This study was approved by the hospital ethics committee (approval number: KYKT2018-002). The consent of each subject was obtained in accordance with the Declaration of Helsinki.

2.2. Data Collection. NIHSS score, onset-to-treatment time (OTT), and blood pressure on admission were collected, as well as demographic data including age, sex, and history of hypertension, diabetes mellitus, smoking, AF, antiplatelet therapy, oral anticoagulant therapy, and previous stroke. Initial counts for white blood cells, neutrophils, lymphocytes, and platelets before IVT were also collected, and NLR was calculated accordingly.

2.3. MRI Analysis. As MRI is more sensitive than CT for detecting HT in AIS [17], we used MRI to confirm and categorize HT. A brain MRI scan was performed for each participant using a 3.0T system (Skyra, Siemens Medical Solutions, Erlangen, Germany) within 3 days after IVT. The sequences of MRI included T1-weighted imaging (T1WI), T2-weighted imaging (T2WI), DWI, and susceptibilityweighted imaging (SWI).

The parameters of each sequence were shown as follows: axial SE T1: time of repetition $(\mathrm{TR})=1500 \mathrm{~ms}$, time of echo $(\mathrm{TE})=11 \mathrm{~ms}$, field of view $(\mathrm{FOV})=220 \mathrm{~mm}$, slice thickness $/$ gap $=4 \mathrm{~mm} / 1.2 \mathrm{~mm}$, and time of acquisition $=1 \min 26 \mathrm{~s}$; Turbo spin echo (TSE) T2: TR $=4720 \mathrm{~ms}, \mathrm{TE}=96 \mathrm{~ms}$, FOV $=220 \mathrm{~mm}$, slice thickness $/ \mathrm{gap}=4 \mathrm{~mm} / 1.2 \mathrm{~mm}$, and time of acquisition $=1 \mathrm{~min} 50 \mathrm{~s}$; DWI: TR $=4640 \mathrm{~ms}$, $\mathrm{TE}=67 \mathrm{~ms}, \quad \mathrm{FOV}=230 \mathrm{~mm}$, slice thickness $/ \mathrm{gap}=4 \mathrm{~mm} /$ $1.2 \mathrm{~mm}$, spin echo planar imaging (EPI) factor $=91$, and acquisition time $=1 \mathrm{~min} 44 \mathrm{~s}$; SWI: TR $=27 \mathrm{~ms}, \mathrm{TE}=20 \mathrm{~ms}$, FOV $=220 \mathrm{~mm}$, slice thickness $/$ gap $=3 \mathrm{~mm} / 0.6 \mathrm{~mm}$, and time of acquisition $=2 \mathrm{~min} 28 \mathrm{~s}$.
HT was defined as the secondary hemorrhage within or away from the infarction area, which appeared as hypointensive lesions on SWI $[18,19]$ and DWI [20]. Calcification was distinguished by CT combined with SWI. As chronic infarction lesions and corresponding old hemorrhage can be detected by T1-weighted imaging (T1WI) and T2-weighted imaging (T2WI), we used these sequences to differentiate acute HT from old hemorrhage.

(1) sHT was defined as NIHSS score increase by $\geq 4$ points compared with that on admission [21].

(2) When HT was positive on MRI, the images were categorized into hemorrhagic infarct (HI) and parenchymatous hemorrhage (PH) according to the ECASS II criteria as follows: HI1, small petechiae along the margins of the infarct; HI2, confluent petechiae within the infarcted area but no space effect; PH1, blood clots in $\leq 30 \%$ of the infarcted area with some slight space-occupying effect; and $\mathrm{PH} 2$, blood clots in $>30 \%$ of the infarcted area with substantial space-occupying effect.

(3) Small vessel disease (SVD) burden was rated on brain MRI by the presence of lacunes, white matter hyperintensities, cerebral microbleeds, and perivascular spaces. The presence of each SVD feature was summed as an "SVD score" (range 0-4) [22].

Two neurologists (D.H.Q. and J.F.Q.) experienced in neuroimaging and trained by a neuroradiologist (M.Q.L.) evaluated the imaging findings for the presence of HT independently, blinded to the patients' clinical information. After observing the images individually, the two observers reviewed all the images to achieve final interobserver consensus.

2.4. Statistical Analysis. Statistical analyses were conducted using SPSS for Windows (v.20.0, IBM Corp., Armonk, NY, USA). Continuous variables with a normal distribution are reported as mean $\pm \mathrm{SD}$, and nonnormally distributed variables as median and interquartile range (IQR). All subjects were divided into two groups based on the presence of HT. Variables were compared using $t$-tests, Mann-Whitney $U$ tests, Pearson $\chi^{2}$ tests, or Fisher's exact tests, as appropriate. Variables with $P<0.05$ in the univariate analysis were included in further binary multivariate logistic regressions. Statistical significance was defined as $P<0.05$ (two-sided).

\section{Results}

During the study period, 306 consecutive patients received IVT with r-tPA within 4.5 hours of stroke onset. In the present study, 21 patients were excluded for the following reasons: additional endovascular therapy after IVT $(n=2)$, permanent or temporary contraindication for MRI $(n=3)$, and no acute lesion found on DWI $(n=16)$. A total of 285 patients were ultimately included.

The average age of the 285 patients was $62.3 \pm 12.0$ years, and $201(70.5 \%)$ patients were male. The median interval between stroke onset and MRI scanning was 42 (range, 
13-65) hours. Antiplatelet agents (aspirin $100 \mathrm{mg} /$ day or clopidogrel $75 \mathrm{mg} /$ day) were prescribed 24 hours after IVT when $\mathrm{PH}$ (7 patients, 2.4\%) was excluded, and no anticoagulants were prescribed during the acute phase. Among the 72 (25.3\%) patients with HT confirmed by MRI, 44 (15.4\%) presented with HI1, 21 (7.4\%) with HI2, 4 (1.4\%) with PH1, and $3(1.1 \%)$ with PH2. Three (1.1\%) patients had sHT. No remote HTs were found in our study. The mean OTT was $200.4 \pm 55.9$ minutes, and the median NIHSS score on admission was 7 (4-10, IQR). The median NLR was 2.700 (1.820-4.255, IQR). High NLR was defined as an NLR value $\geq 4.255$ (75th percentile). Seventy-one (24.9\%) patients had a high NLR before IVT. The demographic and clinical characteristics of this study are shown in Table 1.

3.1. Univariable Analysis. Compared with those without HT, patients with HT had significantly higher NIHSS scores $(P<0.001)$, systolic blood pressure $(\mathrm{SBP})$, platelet counts, lymphocyte counts, and NLR $(P<0.05)$. They also had a greater prevalence of high NLR than those without HT (37.5\% vs. $20.7 \%$ and $P=0.004$ ). Patients with $\mathrm{HT}$ were also more likely to have a history of hypertension and AF. The univariable analysis results are shown in Table 2.

3.2. Multivariate Logistic Regressions. Variables that were significantly different between the two groups in the univariable analysis were entered into subsequent logistic regression model. Since lymphocyte counts and high NLR were highly correlated $(r=-0.499)$, we used two separate logistic regression models. Besides, hypertension and SBP on admission were also highly correlated $(r=0.412)$; therefore, SBP on admission was not included in the regression models to avoid the risk of multicollinearity. In model 1 (with high NLR), NIHSS score on admission (odds ratio $(\mathrm{OR})=1.110$, $95 \%$ confidence interval $(\mathrm{CI})=1.015-1.044$, and $P=0.001$ ), $\mathrm{AF}(\mathrm{OR}=3.986,95 \% \mathrm{CI}=2.095-7.585$, and $P<0.001)$, and high NLR $(\mathrm{OR}=2.078,95 \% \mathrm{CI}=1.078-4.003, P=0.029$, sensitivity 0.375 , and specificity 0.793 ) were significant predictors of HT. In model 2 (with lymphocyte counts), NIHSS score on admission $(\mathrm{OR}=1.111, \quad 95 \%$ $\mathrm{CI}=1.050-1.175$, and $P<0.001)$, $\mathrm{AF}(\mathrm{OR}=3.853,95 \%$ $\mathrm{CI}=2.048-7.248$, and $P<0.001$ ), and lymphocyte counts $(\mathrm{OR}=0.522,95 \% \mathrm{CI}=0.333-0.819$, and $P=0.005)$ were significantly related with HT. Platelet counts and hypertension were not significantly associated with HT in either model. The multivariate logistic regression results for HT risk factors are shown in Table 3.

\section{Discussion}

In our study, high NLR ( $\geq 4.255)$ was significantly associated with HT in AIS patients treated with IVT, which was in accordance with two previous studies $[15,16]$. The mechanism of HT remains uncertain. The disruption of bloodbrain barrier (BBB) and focal inflammation of the infarcted lesion have been reported to be correlated with HT [23]. In accordance with an existing report, neutrophils play a role in $\mathrm{BBB}$ in AIS [24]. Increased neutrophils can result in
Table 1: Demographic and clinical characteristics of the study sample.

\begin{tabular}{|c|c|}
\hline Characteristics & $\begin{array}{c}\text { Mean (SD)/median } \\
(\mathrm{IQR}) / n(\%) \\
(n=285)\end{array}$ \\
\hline Age (years) & $62.3 \pm 12.0$ \\
\hline Men $(n, \%)$ & $201(70.5 \%)$ \\
\hline Hypertension $(n, \%)$ & $211(74.0 \%)$ \\
\hline Diabetes mellitus $(n, \%)$ & $79(27.7 \%)$ \\
\hline Smokers/ex-smokers $(n, \%)$ & $102(35.8 \%)$ \\
\hline Atrial fibrillation $(n, \%)$ & $67(23.5 \%)$ \\
\hline Previous stroke $(n, \%)$ & $52(18.2 \%)$ \\
\hline $\operatorname{PAT}(n, \%)$ & $23(8.1 \%)$ \\
\hline $\operatorname{POAT}(n, \%)$ & $8(2.8 \%)$ \\
\hline \multicolumn{2}{|l|}{ Time of poststroke antiplatelet therapy } \\
\hline Before MRI scan $(n, \%)$ & $212(74.4 \%)$ \\
\hline After MRI scan $(n, \%)$ & $66(23.2 \%)$ \\
\hline No antiplatelet therapy & $7(2.4 \%)$ \\
\hline OTT (minutes) & $200.4 \pm 55.9$ \\
\hline NIHSS score on admission & $7(4-10)^{*}$ \\
\hline Platelet counts $\left(10^{9} / \mathrm{L}\right)$ & $214.0 \pm 55.9$ \\
\hline WBC counts $\left(10^{9} / \mathrm{L}\right)$ & $8.4 \pm 2.9$ \\
\hline Neutrophil counts $\left(10^{9} / \mathrm{L}\right)$ & $5.8 \pm 2.8$ \\
\hline Lymphocyte counts $\left(10^{9} / \mathrm{L}\right)$ & $1.9 \pm 0.9$ \\
\hline NLR & $2.7(1.8-4.3)^{*}$ \\
\hline High NLR $(n, \%)$ & $71(24.9 \%)$ \\
\hline Uric acid $(\mathrm{mmol} / \mathrm{L})$ & $390.1 \pm 108.6$ \\
\hline $\mathrm{BG}$ on admission $(\mathrm{mmol} / \mathrm{L})$ & $7.5 \pm 3.2$ \\
\hline SBP on admission $(\mathrm{mmHg})$ & $157.4 \pm 24.8$ \\
\hline DUB on admission (mmHg) & $91.4 \pm 16.5$ \\
\hline SVD burden & $1(0-2)^{*}$ \\
\hline Hemorrhagic transformation $(n, \%)$ & $72(25.3 \%)$ \\
\hline HI1 & $44(15.4 \%)$ \\
\hline HI2 & $21(7.4 \%)$ \\
\hline $\mathrm{PH} 1$ & $4(1.4)$ \\
\hline $\mathrm{PH} 2$ & $3(1.1 \%)$ \\
\hline $\operatorname{sHT}(n, \%)$ & $3(1.1 \%)$ \\
\hline
\end{tabular}

$\mathrm{BG}=$ blood glucose; $\mathrm{DBP}=$ diastolic blood pressure; $\mathrm{HI}=$ hemorrhagic infarct; NIHSS=National Institutes of Health Stroke Scale; NLR=neutrophil-to-lymphocyte ratio; OTT $=$ onset-to-treatment time; $\mathrm{PAT}=$ previous antiplatelet therapy; $\mathrm{PH}=$ parenchymatous hemorrhage; $\mathrm{POAT}=$ previous oral anticoagulant therapy; $\mathrm{SBP}=$ systolic blood pressure; $\mathrm{sHT}=$ symptomatic hemorrhagic transformation; SVD $=$ small vessel disease; $\mathrm{WBC}=$ white blood cell * median (25Q-75Q).

enhanced expression of matrix metalloproteinase-9 [25], which has been linked to BBB damage and HT in AIS patients [26-28]. Lymphocytes play important roles in inflammation $[29,30]$. However, the precise effects depend on the subtype of lymphocytes. Some are neuroprotective $[31,32]$, while others exacerbate inflammation [33, 34]. A high NLR value represents high neutrophil counts and/or low lymphocyte counts. NLR is considered a good marker that simultaneously reflects the negative effects of neutrophils and positive effects of lymphocytes in stroke patients $[35,36]$. High NLR was found to predict poor outcomes of AIS patients [37-39]. In our study, both lymphocyte counts and high NLR were significantly associated with HT in logistic regression analyses. However, absolute lymphocyte counts vary among individuals, even in healthy subjects. Thus, NLR may be a more stable and suitable marker than absolute lymphocyte counts for predicting HT. Neutrophil 
TABLE 2: Comparisons of clinicial and laboratory variables in IVT patients with and without HT.

\begin{tabular}{|c|c|c|c|c|}
\hline Variable & $\begin{array}{l}\text { With HT } \\
(N=72)\end{array}$ & $\begin{array}{c}\text { Without HT } \\
(N=213)\end{array}$ & $\begin{array}{l}t / X^{2} / z \\
\text { value }\end{array}$ & $P$ value \\
\hline Age $^{a}$ (years) & $64.0 \pm 12.3$ & $61.8 \pm 11.9$ & -1.354 & 0.177 \\
\hline Men $^{\mathrm{b}}$ & $51(70.8 \%)$ & $150(70.4 \%)$ & 0.004 & 0.947 \\
\hline NIHSS score on admission ${ }^{c}$ & $10(6-14.8)$ & $6(4-9)$ & -5.429 & $<0.001$ \\
\hline Hypertension $^{\mathrm{b}}$ & $46(63.9 \%)$ & $165(77.5 \%)$ & 5.159 & 0.023 \\
\hline Diabetes $^{\mathrm{b}}$ & $19(26.4 \%)$ & $60(28.2 \%)$ & 0.085 & 0.770 \\
\hline Smokers ${ }^{\mathrm{b}}$ & $25(34.7 \%)$ & $77(36.2 \%)$ & 0.048 & 0.827 \\
\hline $\mathrm{AF}^{\mathrm{b}}$ & $35(48.6 \%)$ & $32(15.0 \%)$ & 33.759 & $<0.001$ \\
\hline Previous stroke $\mathrm{e}^{\mathrm{b}}$ & $13(18.1 \%)$ & $39(18.3 \%)$ & 0.002 & 0.961 \\
\hline $\mathrm{PAT}^{\mathrm{b}}$ & $3(4.2 \%)$ & $20(9.4 \%)$ & 1.979 & 0.213 \\
\hline $\mathrm{POAT}^{\mathrm{b}}$ & $3(4.2 \%)$ & $5(2.3 \%)$ & 0.653 & 0.421 \\
\hline Antiplatelet therapy prescribed before MRI scan ${ }^{\mathrm{b}}$ & $45(62.5 \%)$ & $167(78.4 \%)$ & 2.315 & 0.128 \\
\hline Uric $\operatorname{acid}^{\mathrm{a}}(\mathrm{mmol} / \mathrm{L})$ & $369.2 \pm 108.8$ & $396.9 \pm 107.9$ & 1.844 & 0.066 \\
\hline $\mathrm{OTT}^{\mathrm{a}}$ (minutes) & $195.3 \pm 52.6$ & $202.2 \pm 56.9$ & 0.898 & 0.37 \\
\hline Platelet counts ${ }^{\mathrm{a}}\left(10^{9} / \mathrm{L}\right)$ & $201.3 \pm 43.2$ & $218.3 \pm 59.0$ & 2.246 & 0.025 \\
\hline $\mathrm{WBC}$ counts $^{\mathrm{a}}\left(10^{9} / \mathrm{L}\right)$ & $8.6 \pm 3.2$ & $8.4 \pm 2.8$ & -0.586 & 0.558 \\
\hline Neutrophil counts $\left(10^{9} / \mathrm{L}\right)$ & $6.3 \pm 3.3$ & $5.6 \pm 2.6$ & -1.759 & 0.08 \\
\hline Lymphocyte counts $\left(10^{9} / \mathrm{L}\right)$ & $1.6 \pm 0.7$ & $2.1 \pm 0.9$ & 4.349 & $<0.001$ \\
\hline High NLR ${ }^{c}$ & $27(37.5 \%)$ & $44(20.7 \%)$ & 8.16 & 0.004 \\
\hline $\mathrm{BG}$ on admission ${ }^{\mathrm{a}}(\mathrm{mmol} / \mathrm{L})$ & $7.6 \pm 3.4$ & $7.5 \pm 3.2$ & -0.280 & 0.779 \\
\hline SBP on admission ${ }^{\mathrm{a}}(\mathrm{mmHg})$ & $152.0 \pm 24.1$ & $159.2 \pm 24.8$ & 2.135 & 0.035 \\
\hline DBP on admission ${ }^{\mathrm{a}}(\mathrm{mmHg})$ & $90.1 \pm 15.0$ & $91.8 \pm 17.0$ & 0.754 & 0.452 \\
\hline SVD burden ${ }^{c}$ & $0(0-1)$ & $1(0-2)$ & -1.434 & 0.152 \\
\hline
\end{tabular}

${ }^{a}$ Mean (SD), $t$-test; ${ }^{\mathrm{b}} n$ (\%), chi-square test; ${ }^{\mathrm{c}}$ Mann-Whitney $U$ test. $\mathrm{AF}=$ atrial fibrillation; $\mathrm{BG}=$ blood glucose; $\mathrm{DBP}=$ diastolic blood pressure; $\mathrm{HTh}=\mathrm{e}-$ morrhagic transformation; NIHSS = National Institutes of Health Stroke Scale; NLR = neutrophil-to-lymphocyte ratio; OTT =onset-to-treatment time; $\mathrm{PAT}=$ previous antiplatelet therapy; $\mathrm{POAT}=$ previous oral anticoagulant therapy; $\mathrm{SBP}=$ systolic blood pressure; $\mathrm{SVD}=$ small vessel disease; $\mathrm{WBC}=$ white blood cell.

TABle 3: Multivariate logistic regression of risk factors for hemorrhagic transformation.

\begin{tabular}{lccc}
\hline Variable & $\beta$ & OR $(95 \% \mathrm{CI})$ & $P$ value \\
\hline Model 1 (with high NLR entered) & & & \\
NIHSS score on admission & 0.100 & $1.110(1.015-1.044)$ & 0.001 \\
Hypertension & -0.399 & $0.671(0.352-1.280)$ & 0.226 \\
AF & 1.383 & $3.986(2.095-7.585)$ & $0.995(0.988-1.001)$ \\
Platelet counts & -0.006 & $2.078(1.078-4.003)$ & 0.078 \\
High NLR & 0.731 & & 0.029 \\
Model 2 (with lymphocyte counts entered) & & $1.111(1.050-1.175)$ & $<0.001$ \\
NIHSS score on admission & 0.105 & $0.644(0.336-1.237)$ & 0.181 \\
Hypertension & -0.440 & $3.853(2.048-7.248)$ & $<0.001$ \\
AF & 1.349 & $0.996(0.990-1.003)$ & 0.240 \\
Platelet counts & -0.004 & $0.522(0.333-0.819)$ \\
Lymphocyte counts & 0.650 & 0.005 \\
\hline
\end{tabular}

$\mathrm{AF}=$ atrial fibrillation; NIHSS = National Institutes of Health Stroke Scale; NLR = neutrophil-to-lymphocyte ratio.

counts were not significant in the univariable analysis. However, since neutrophil counts were highly related with NLR $(r=0.676)$, our findings did not contradict previous studies. NLR is easily evaluated with a routine blood test, making it an economic and effective marker, even in regional hospitals.

There were several advantages to our study. First, to the best of our knowledge, it was one of the few that focused on the association between high NLR and HT in IVT-treated AIS patients. Second, all the participants had relatively complete data of neuroimaging including SWI. However, our results should be considered in the context of several limitations. First, repeated MR scanning was not performed, which might have led to underestimation of HT in the subacute phase. Second, we lacked dynamic NLR data, which would be considered a more effective predictor. Third, the numbers of cases with $\mathrm{PH} 1, \mathrm{PH} 2$, or sHT were too small to perform further analyses of these severe HT subtypes.

\section{Conclusion}

High NLR was a useful predictor of HT in AIS patients after IVT. Further prospective studies with larger sample sizes, repeated MR scans, and dynamic NLR are warranted.

\section{Data Availability}

The data used to support the findings of the study are available from the corresponding author upon request. 


\section{Conflicts of Interest}

The authors declare no conflicts of interest.

\section{Authors' Contributions}

Y. L. L. and Y. K. C. participated in the conception and design of the study, the analysis of clinical data, and critical revision of the manuscript for scientific validity. D. H. Q., J. F. Q., and M. Q. L. analyzed the imaging data. J. K. L., H. P. Y., and P. S. X. helped to acquire raw data. All authors have read and approved the final manuscript.

\section{Acknowledgments}

This study was supported by the Medical Scientific Research Foundation of Guangdong Province, China (No. A2018322).

\section{References}

[1] N. Listed, "The National Institute of Neurological Disorders and Stroke RT-PA stroke study Group. Tissue plasminogen activator for acute ischemic stroke," The New England Journal of Medicine, vol. 334, no. 1405, p. 1406, 1996.

[2] H. Werner, K. Markku, B. Erich et al., "Thrombolysis with alteplase 3 to 4.5 hours after acute ischemic stroke," The New England Journal of Medicine, vol. 359, pp. 1317-1329, 2008.

[3] R. I. Lindley, J. M. Wardlaw, P. A. G. Sandercock et al., "Frequency and risk factors for spontaneous hemorrhagic transformation of cerebral infarction," Journal of Stroke and Cerebrovascular Diseases, vol. 13, no. 6, pp. 235-246, 2004.

[4] T. Valeria, D. Marco, D. B. Norma et al., "Frequency and determinants for hemorrhagic transformation of cerebral infarction," Neuroepidemiology, vol. 33, pp. 261-265, 2009.

[5] L. A. Beslow, S. E. Smith, A. Vossough et al., "Hemorrhagic transformation of childhood arterial ischemic stroke," Stroke, vol. 42, no. 4, pp. 941-946, 2011.

[6] D. Strbian, T. Sairanen, A. Meretoja et al., "Patient outcomes from symptomatic intracerebral hemorrhage after stroke thrombolysis," Neurology, vol. 77, no. 4, pp. 341-348, 2011.

[7] K. R. Lees, E. Bluhmki, R. von Kummer et al., "Time to treatment with intravenous alteplase and outcome in stroke: an updated pooled analysis of ECASS, ATLANTIS, NINDS, and EPITHET trials," The Lancet, vol. 375, no. 9727, pp. 1695-1703, 2010.

[8] R. C. S. Seet, "Relationship between chronic atrial fibrillation and worse outcomes in stroke patients after intravenous thrombolysis," Archives of Neurology, vol. 68, no. 11, p. 1454, 2011.

[9] R. Yue, D. Li, J. Yu et al., "Atrial fibrillation is associated with poor outcomes in thrombolyzed patients with acute ischemic stroke: a systematic review and Meta-Analysis," Medicine, vol. 95, Article ID e3054, 2016.

[10] C.-T. Ong, Y.-S. Wong, C.-S. Wu, and Y.-H. Su, "Outcome of stroke patients receiving different doses of recombinant tissue plasminogen activator," Drug Design, Development and Therapy, vol. 11, pp. 1559-1566, 2017.

[11] A. M. Demchuk, L. B. Morgenstern, D. W. Krieger et al., "Serum glucose level and diabetes predict tissue plasminogen activator-related intracerebral hemorrhage in acute ischemic stroke," Stroke, vol. 30, no. 1, pp. 34-39, 1999.

[12] L. Willer, I. Havsteen, C. Ovesen, A. F. Christensen, and H. Christensen, "Computed tomography-verified leukoaraiosis is a risk factor for post-thrombolytic hemorrhage," Journal of Stroke and Cerebrovascular Diseases, vol. 24, no. 6, pp. 11261130, 2015.

[13] M. Mazya, J. A. Egido, G. A. Ford et al., "Predicting the risk of symptomatic intracerebral hemorrhage in ischemic stroke treated with intravenous alteplase," Stroke, vol. 43, no. 6, pp. 1524-1531, 2012.

[14] K. Liu, S. Yan, S. Zhang, Y. Guo, and M. Lou, "Systolic blood pressure variability is associated with severe hemorrhagic transformation in the early stage after thrombolysis," Translational Stroke Research, vol. 7, no. 3, pp. 186-191, 2016.

[15] Z. Guo, S. Yu, L. Xiao et al., "Dynamic change of neutrophil to lymphocyte ratio and hemorrhagic transformation after thrombolysis in stroke," Journal of Neuroinflammation, vol. 13, p. 199, 2016.

[16] Q. Song, Y. Li, Y. Wang, C. Wei, J. Liu, and M. Liu, "Increased neutrophil-to-lymphocyte ratios are associated with greater risk of hemorrhagic transformation in patients with acute ischemic stroke," Current Neurovascular Research, vol. 15, pp. 326-335, 2018.

[17] M. C. Arnould, C. A. Grandin, G. Cosnard, and T. P. Duprez, "Comparison of CT and three MR sequences for detecting and categorizing early (48 hours) hemorrhagic transformation in hyperacute ischemic stroke," American Journal of Neuroradiology, vol. 25, pp. 939-944, 2004.

[18] N. D. Wycliffe, J. Choe, B. Holshouser, U. E. Oyoyo, E. M. Haacke, and D. K. Kido, "Reliability in detection of hemorrhage in acute stroke by a new three-dimensional gradient recalled echo susceptibility-weighted imaging technique compared to computed tomography: a retrospective study," Journal of Magnetic Resonance Imaging, vol. 20, no. 3, pp. 372-377, 2004.

[19] A. Masuma, H. Toshinori, H. Yasuhiro et al., "Detection of hemorrhagic hypointense foci in the brain on susceptibilityweighted imaging clinical and phantom studies," Academic Radiology, vol. 14, pp. 1011-1019, 2007.

[20] T. Ebisu, C. Tanaka, M. Umeda et al., "Hemorrhagic and nonhemorrhagic stroke: diagnosis with diffusion-weighted and T2-weighted echo-planar MR imaging," Radiology, vol. 203, no. 3, pp. 823-828, 1997.

[21] W. Hacke, M. Kaste, C. Fieschi et al., "Randomised doubleblind placebo-controlled trial of thrombolytic therapy with intravenous alteplase in acute ischaemic stroke (ECASS II)," The Lancet, vol. 352, no. 9136, pp. 1245-1251, 1998.

[22] S. Julie, S. D. J. Makin, F. N. Doubal, M. S. Dennis, and J. M. Wardlaw, "Stroke subtype, vascular risk factors, and total MRI brain small-vessel disease burden," Neurology, vol. 83, pp. 1228-1234, 2014.

[23] G. C. Jickling, B. P. Ander, S. Boryana et al., "RNA in blood is altered prior to hemorrhagic transformation in ischemic stroke," Annals of Neurology, vol. 74, pp. 232-240, 2013.

[24] G. C. Jickling, D. Liu, B. P. Ander, B. Stamova, X. Zhan, and F. R. Sharp, "Targeting neutrophils in ischemic stroke: translational insights from experimental studies," Journal of Cerebral Blood Flow \& Metabolism, vol. 35, no. 6, pp. 888-901, 2015.

[25] J. Carles, P. Julián, S Sònia et al., "Neutrophil infiltration increases matrix metalloproteinase- 9 in the ischemic brain after occlusion/reperfusion of the middle cerebral artery in rats," J Cereb Blood Flow Metab, vol. 23, pp. 1430-1440, 2003.

[26] G. C. Jickling, D. Liu, B. Stamova et al., "Hemorrhagic transformation after ischemic stroke in animals and humans," Journal of Cerebral Blood Flow \& Metabolism, vol. 34, no. 2, pp. 185-199, 2014. 
[27] L. Zhang, Z. G. Zhang, R. L. Zhang, M. Lu, M. Krams, and M. Chopp, "Effects of a selective CD11b/CD18 antagonist and recombinant human tissue plasminogen activator treatment alone and in combination in a rat embolic model of stroke," Stroke, vol. 34, no. 7, pp. 1790-1795, 2003.

[28] A. Rosell, E. Cuadrado, A. Ortega-Aznar, M. HernándezGuillamon, E. H. Lo, and J. Montaner, "MMP-9-Positive neutrophil infiltration is associated to blood-brain barrier breakdown and basal lamina type IV collagen degradation during hemorrhagic transformation after human ischemic stroke," Stroke, vol. 39, no. 4, pp. 1121-1126, 2008.

[29] M. Schwartz and G. Moalem, "Beneficial immune activity after CNS injury: prospects for vaccination," Journal of Neuroimmunology, vol. 113, no. 2, pp. 185-192, 2001.

[30] N. Frangogiannis, C. W. Smith, and M. L. Entman, "The inflammatory response in myocardial infarction," Cardiovascular Research, vol. 53, no. 1, pp. 31-47, 2002.

[31] R. Xuefang, A. Kozaburo, D. Suzan et al., "Regulatory B cells limit CNS inflammation and neurologic deficits in murine experimental stroke," Journal of Neuroscience, vol. 31, pp. 8556-8563, 2011.

[32] A. Liesz, W. Zhou, S.-Y. Na et al., "Boosting regulatory t cells limits neuroinflammation in permanent cortical stroke," Journal of Neuroscience, vol. 33, no. 44, pp. 17350-17362, 2013.

[33] J. Y. Kim, M. Kawabori, and M. A. Yenari, "Innate inflammatory responses in stroke: mechanisms and potential therapeutic targets," Current Medicinal Chemistry, vol. 21, pp. 2076-2097, 2014.

[34] A. Liesz, X. Hu, C. Kleinschnitz, and H. Offner, "Functional role of regulatory lymphocytes in stroke: facts and controversies," Stroke, vol. 46, no. 5, pp. 1422-1430, 2015.

[35] P. H. Gibson, B. H. Cuthbertson, B. L. Croal et al., "Usefulness of Neutrophil/Lymphocyte ratio as predictor of New-Onset atrial fibrillation after coronary artery bypass grafting," The American Journal of Cardiology, vol. 105, no. 2, pp. 186-191, 2010.

[36] S. Gökhan, A. Ozhasenekler, D. H. Mansur, E. Akil, M. Ustündag, and M. Orak, "Neutrophil lymphocyte ratios in stroke subtypes and transient ischemic attack," European Review for Medical and Pharmacological Sciences, vol. 17, pp. 653-657, 2013.

[37] S. Yu, H. Arima, C. Bertmar, S. Clarke, G. Herkes, and M. Krause, "Neutrophil to lymphocyte ratio and early clinical outcomes in patients with acute ischemic stroke," Journal of the Neurological Sciences, vol. 387, pp. 115-118, 2018.

[38] S. Qun, Y. Tang, J. Sun et al., "Neutrophil-To-Lymphocyte ratio predicts 3-Month outcome of acute ischemic stroke," Neurotoxicity Research, vol. 31, no. 3, pp. 444-452, 2017.

[39] J. Xue, W. Huang, X. Chen et al., "Neutrophil-to-Lymphocyte ratio is a prognostic marker in acute ischemic stroke," Journal of Stroke and Cerebrovascular Diseases, vol. 26, no. 3, pp. 650-657, 2017. 